Dramenmodell und ideologische Entwicklung der italienischen Oper im frühen Ottocento 
Fabian A. Stallknecht

\section{Dramenmodell und}

ideologische Entwicklung

der italienischen Oper

im frühen Ottocento

Verlag J. B. Metzler

Stuttgart - Weimar 
Die Deutsche Bibliothek - CIP-Einheitsaufnahme

\author{
Stallknecht, Fabian A.: \\ Dramenmodell und ideologische Entwicklung der italienischen Oper \\ im frühen Ottocento / Fabian A. Stallknecht. \\ - Stuttgart ; Weimar : Metzler, 2001 \\ ISBN 978-3-476-45251-1 \\ ISBN 978-3-476-02753-5 (eBook) \\ DOI 10.1007/978-3-476-02753-5
}

Dieses Werk einschließlich aller seiner Teile ist urheberrechtlich geschützt. Jede Verwertung außerhalb der engen Grenzen des Urheberrechtsgesetzes ist ohne Zustimmung des Verlages unzulässig und strafbar. Das gilt insbesondere für Vervielfältigungen, Übersetzungen, Mikroverfilmungen und die Einspeicherung und Verarbeitung in elektronischen Systemen.

M \& P Schriftenreihe für Wissenschaft und Forschung

(C) 2001 Springer-Verlag GmbH Deutschland

Ursprünglich erschienen bei J.B.Metzlersche Verlagsbuchhandlung und Carl Ernst Poeschel Verlag GmbH in Stuttgart 2001 


\section{INHALTSÜBERSICHT:}

Seite

Vorwort

I.1 Napoléon und das napoleonische Regierungssystem in Italien.

I.2 Die Situation Italiens innerhalb der Neuordnung Europas.

I.3 Die Rolle des italienischen Bürgertums und die Entstehung der Società segrete.

I.4 Die gescheiterte Revolution von 1820/21 und ihre gesellschaftlichen Konsequenzen.

I.5 Die Julirevolution in Frankreich und ihre Auswirkungen auf die italienische Einigungsbewegung.

II. Die italienische Romantik und das geistig-kulturelle

Klima Italiens in der ersten Hälfte des 19. Jahrhunderts.

III. Das Musikleben Italiens, das Theatersystem im 19. Jahrhundert und die kompositorisch-stilistische Entwicklung der italienischen Oper zwischen belcanto und romanticismo.

IV. Die Opern Rossinis vor 1815. Tancredi.

V. Die neapolitanischen Opern Rossinis 1818-22. Mosè in

Egitto, La donna del lago, Maometto II.

VI. Die Pariser Opern Rossinis 1825-29. Il Viaggio a Reims, Le Siège de Corinthe, Moise et Pharaon, Guillaume Tell.

VII. Die Opern Bellinis und Donizettis vor 1830. Il Pirata, La Straniera, I Capuleti e i Montecchi, Anna Bolena.

VIII. Die Opern Bellinis nach 1830. La Sonnambula, Norma, I Puritani.

IX. Die Opern Donizettis nach 1830. Lucrezia Borgia, Lucia di Lammermoor, Maria Stuarda, Roberto Devereux. 


\section{VORWORT}

Wir alten eingefleischten Wagnerianer sind doch die dankbarsten Belliniund Rossini-Hörer. Friedrich Nietzsche ${ }^{1}$

Wenn Friedrich Lippmann 1983 in einem Programmheftbeitrag einleitend bemerkt „Das Bild Bellinis schwankt in der Geschichte“"2, so läßt sich diese Feststellung auch auf das Oeuvre Gioachino ${ }^{3}$ Rossinis und Gaëtano Donizettis ausdehnen. Wenn man die heutige Rezeption ihrer Werke aus einem kritischen Blickwinkel betrachtet, so bietet sich eine überaus ambivalente Situation, die gleichermaßen zu Freude und Bedenken Anlaß gibt. Erfreulich ist ohne Zweifel die Tatsache, daß das Opernschaffen des späten 18. und frühen 19. Jahrhunderts in den letzten ca. vierzig Jahren eine stetige und, so hat es den Anschein, unaufhaltsame Popularisierung erfahren hat, nachdem das Publikumsinteresse an den Werken der genannten Komponisten gegen Ende des 19. Jahrhunderts dramatisch abgesunken war und alle drei nur noch mit wenigen populären Titeln wie Il Barbiere di Siviglia ${ }^{4}$ oder Lucia di Lammermoor im öffentlichen Bewußtsein präsent waren. Die Wiederentdeckung zunächst Bellinis und Donizettis, mit geringfügiger Verzögerung auch diejenige Rossinis, nahm in den fünfziger Jahren ihren Anfang und ereignete sich gleichermaßen auf publizistischem 'zitiert nach: Programmheft der Wiener Staatsoper zu I Puritani. Wien 1994, Seite 10

${ }^{2}$ Lippmann Der Komponist der „Norma“. In: Programmheft der Bonner Oper zu Norma. Bonn 1983, Seite 11

${ }^{3}$ Rossini selbst verwendet in Briefen und anderen Dokumenten unterschiedliche Schreibweisen seines Vormamens, in der Literatur hat sich inzwischen diejenige mit einem „c“ eingebürgert, welche auch in der vorliegenden Abhandlung Verwendung findet. Abweichende Schreibweisen in zitierten Textpassagen bleiben davon unberührt.

${ }^{4}$ Der geringe Bekanntheitsgrad Rossinis bezieht sich hauptsächlich auf den seria- Komponisten. Die turbulenten musikalischen Komödien wie der genannte Barbiere, La Cenerentola oder L'Italiana in Algeri haben hingegen eine lückenlose Rezeptionsgeschichte und erfreuten sich zu allen Zeiten großer Beliebtheit. 
fang und ereignete sich gleichermaßen auf publizistischem wie auf aufführungspraktischen Gebiet, 1959 veröffentlichte Francesco Pastura die erste Bellini- Monographie mit wissenschaftlichem Anspruch. Zugleich fanden die Opern des Belcanto und Romanticismo durch die theaterpraktischen Pioniertaten von Künstlern wie Lucchino Visconti, Gianandrea Gavazzeni oder Maria Callas allmählich Einlaß ins Repertoire bedeutender Opernhäuser, zunächst in Italien, später auch in anderen europäischen Ländern. Heute läßt sich mit Recht und Fug behaupten, daß die Werke Rossinis, Bellinis und Donizettis einen zwar nicht beherrschenden, aber zumindest einen festen Platz im Musik- und Theaterbetrieb unserer Tage einnehmen. Daß inzwischen sämtliche Opern von Bellini und die weitaus meisten von Rossini und Donizetti in zum Teil konkurrierenden Gesamteinspielungen vorliegen und auch weniger bekannte Titel mit einer gewissen Regelmäßigkeit auf den Spielplänen erscheinen, mag als Beleg für meine These gelten.

Der problematische Aspekt dieser an sich begrüßenswerten Entwicklung ist, daß sie in wesentlichen Punkten auf einem fundamentalen Mißverständnis basiert. Dessen Hintergründe sind außerordentlich komplex und beinhalten theatergeschichtliche, stilistische, ästhetische und aufführungspraktische Aspekte, welche an dieser Stelle nur angedeutet werden können. Das Kernproblem besteht zum einen in der Reduzierung der Opern Rossinis, Bellinis und Donizettis auf ihre rein wirkungsästhetische Qualität, das sinnliche Faszinosum und den hedonistischen Reiz vokaler Bravourakte, und zum anderen in der Ablösung der musikdramatischen Konzeption des romantischen Melodramma von seinen historischen, geistesgeschichtlichen und ideologischen Hintergründen. Indem Interpreten, Kommentatoren und Rezipienten 
die gesellschaftliche Rolle und die politischen und ideologischen Implikationen des Melodramma negiert haben, haben sie die italienische Oper insgesamt, und diejenige vor Verdi im Besonderen, zu einem klingenden Konsumartikel degradiert, zu einem „Synonym für begriffslose musikalische Schönheit" ${ }^{* 5}$, wie Döhring schreibt. Über die Hintergründe einer solchen Verfälschung läßt sich nur spekulieren, doch seien zumindest zwei Gesichtspunkte erwähnt: zum einen haben aufführungspraktische Manipulationen wie Transpositionen, Striche, insbesondere das verbreitete Weglassen von Cabalettenreprisen, und eine stilistische Indifferenz der musikalischen Interpretation in den letzten Jahrzehnten ein fragwürdiges Bild von der kompositorischen und musikdramatischen Gestalt dieser Opern vermittelt, und zum anderen hat die repertoirebeherrschende Position der Werke Giuseppe Verdis ${ }^{6}$ und Giacomo Puccinis zu einer Verschiebung von Rezeptionskriterien geführt. Verdis musikalisches Idiom und vor allem seine patriotischen Chorhymnen wurden zum fanalartigen Inbegriff der Politisierung und Ideologisierung des italienischen Musiktheaters, vor deren Rigorosität und klanglichen Vergröberung die musikdramatischen Konzepte der vorherigen Epoche zwangsläufig verblassen mußten.

Auf diese Weise hat sich im Laufe der Zeit ein irriges, von sentimentalen Klischees verzerrtes Verständnis dieser Komponistengeneration und ihres Schaffens herausgebildet, welches durch einen nahezu unerschöpflichen Vorrat liebenswerter, der sachlichen Überprüfung aber in den wenigsten Fällen standhaltender, Anekdoten noch zusätzlich ausgeschmückt und ver-

\footnotetext{
${ }^{5}$ Sieghart Döhring „Lucia di Lammermoor": Stationendrama eines Untergangs. In: Programmheft der Bayerischen Staatsoper zu Lucia di Lammermoor. München 1991, Seite 87

${ }^{6}$ Es sei allerdings einschränkend hinzugefügt, daß einem breiten Publikum nur ein Bruchteil der 32 Opern Verdis bekannt ist.
} 
festigt worden ist. Unter Berufung auf prominente, gleichwohl selten unvoreingenommene Zeitgenossen wie Heinrich Heine oder Richard Wagner und unter dem Eindruck des nördlich der Alpen bewußtseinsbildenden Geniekults der Spätromantik hat es sich eingebürgert, in Bellini einen melancholisch- elegischen „Mondschein-Musiker“" , in Donizetti den „Maestrorgasmo ${ }^{\text {" } 8}$, den spukhaft-erotisierten, stets am Rande der geistigen Paralyse entlang balancierenden Schauerromantiker oder in Rossini einen humorvolljovialen Gourmet und Schöngeist zu sehen. Zweifellos hat Rossini besagtes Klischee im Sinne einer beinahe neu zeitlich anmutenden „Imagebildung“ selbst nach Kräften verbreitet und ebenso zweifellos haben sich die tatsächlich tragischen, mit dem Ruch schicksalhafter und geheimnisvoller Fügung behafteten Lebensumstände Bellinis und Donizettis für die beschriebene Mythenbildung als förderlich erwiesen, dennoch haben solche Vorstellungen nur wenig mit der historischen und künstlerischen Realität gemein.

Unter diesen Umständen verwundert es nicht, daß die gesellschaftliche, soziale und ideologische Bedeutung des Musiktheaters im frühen Ottocento und die seiner führenden Protagonisten in der historischen und theaterwissenschaftlichen Forschung lange Zeit unbeachtet geblieben ist. Zwar haben verschiedene Autoren gegen Ende des 19. Jahrhunderts den Versuch unternommen, das Werk vor allem Rossinis und Bellinis in Beziehung zum Risorgimento zu setzen, doch bieten diese Ansätze weder ein konsequentes Bild noch eine sinnfällige, analytisch fundierte Begründung. In den meisten Publikationen des zwanzigsten Jahrhunderts werden die politischen und

\footnotetext{
${ }^{7}$ R.Steiner-Isenmann: Gaëtano Donizetti. Sein Leben und seine Opern. Bern/Stuttgart 1982, Seite 98 ${ }^{8}$ Diesen anschaulichen Beinamen erwarb sich der Komponist sowohl wegen seines Lebenswandels, als auch aufgrund der für zeitgenössische Hörgewohnheiten oftmals brutalen Leidenschaftlichkeit seiner Klangsprache. Vergl.: William Ashbrook: Donizetti and his operas. London 1965, Seite 147
} 
ideologischen Implikationen im Oeuvre Rossinis, Bellinis und Donizettis hingegen ausgeklammert oder sogar explizit bestritten. Erst in den letzten Jahren sind die politische Interpretierbarkeit und die ideologische Konzeption zumindest der Opern Rossinis stärker ins Blickfeld der Forschung gerückt, nach den quantitativ auf Essayformat beschränkten Beiträgen von Schläder ${ }^{9}$ und Gossett ${ }^{10}$ hat Grempler ${ }^{11}$ im Jahr 1996 die erste umfassende Untersuchung über Rossinis Verhältnis zur Politik und die politische Deutung seiner Opern vorgelegt; eine Publikation, die aufgrund der Fülle und des Detailreichtums des präsentierten Materials von großem Interesse ist, die Thematik jedoch etwas einseitig auf tagespolitische Fragestellungen hin behandelt. Im Falle Bellinis und Donizettis ist eine vergleichbare Arbeit bis heute nicht geleistet worden und auch eine Gesamtdarstellung dieser Komponistengeneration und der ideologischen Aspekte ihres Schaffens fehlt nach wie vor.

Die vorliegende Abhandlung versteht sich als Versuch, diese publizistische Lücke zu schließen und das musikalische Theater Rossinis, Bellinis und Donizettis anhand ausgewählter Werke aller drei Komponisten in Beziehung zu den historischen, gesellschaftspolitischen, geistes- und mentalitätsgeschichtlichen und weltanschaulichen Entwicklungen der Zeit zu setzen und die analysierten Opern als Ausdruck eines von deprimierenden Erfahrungen geprägten Lebensgefühls und Gesellschaftsbildes und als ge-

\footnotetext{
"Jürgen Schläder: ,....stark von revolutionären Gedanken beeinflußt. “ Über die politische Dimension in Gioacchino Rossinis Opern. In: Jahrbuch der Bayerischen Staatsoper 1992/93, hrsg. von H.Krellmann. München 1992

${ }^{10}$ Philip Gossett: Reactionary or Patriot? A Rossinian Dilemma. In: Programmheft des Rossini Opera Festival zu Pensa alla patria. Pesaro 1994

${ }^{11}$ Martina Grempler: Rossini e la patria. Studien zu Leben und Werk Gioachino Rossinis vor dem Hintergrund des Risorgimento. Kassel 1996
} 
schichtsmächtige Zeugnisse einer politisch und geistesgeschichtlich bewegten Epoche zu interpretieren. Aus diesem Grund nimmt die Darstellung der historischen und politischen Zusammenhänge, des kulturellen und weltanschaulichen Klimas der Zeit sowie der konkreten Arbeits- und Produktionsbedingungen in der vorliegenden Abhandlung einen weitaus größeren Raum ein als in theaterwissenschaftlichen Arbeiten üblich. Der hohe Komplexionsgrad der behandelten Kunstwerke macht es unmöglich, diese im Rahmen einer Abhandlung wie der vorliegenden wirklich erschöpfend, d.h. in allen für ihre ästhetische Wirkung und musikhistorische Bedeutung relevanten Aspekten zu analysieren und zu würdigen, meine Themenstellung bedeutet a priori eine selektive Betrachtung der politisch- ideologischen, gesellschaftlichen und geistesgeschichtlichen Implikationen. Quellenkundliche, stoffgeschichtliche und musikologische Erwägungen können daher lediglich eine untergeordnete Rolle spielen und werden nur soweit in die Untersuchung einbezogen, wie die Themenstellung dies erfordert. Die Sichtung der vorhandenen Sekundärliteratur über Leben und Werk der drei Komponisten hat, von wenigen Ausnahmen abgesehen, in erster Linie deren Irrelevanz für die behandelte Themenstellung ergeben, auf eine zusammenfassende Darstellung der dort über weite Strecken dokumentierten Klischeevorstellungen und Missverständnisse habe ich bewußt verzichtet und diesen Aspekt auf wenige exemplarische Textzitate beschränkt. Schriftliche Selbstzeugnisse der Autoren werden ebenfalls nur in geringer Quantität berücksichtigt, da solche naturgemäß ein hohes $\mathrm{Ma} \beta$ an momentanen Stimmungen und wandelbaren Empfindungen transportieren und daher rein subjektiven Charakter haben; im Falle Gioachino Rossinis konstituieren diese sogar ein nicht eben vorteilhaftes, von Unehrlichkeit, Berechnung und Op- 
portunismus geprägtes Charakterbild. Die Grundlage der vorliegenden Untersuchungen und Analysen bilden vielmehr die Libretti und Notentexte der ausgewählten Opern in Relation zu deren geistesgeschichtlichem und gesellschaftlichem Kontext, wie er sich uns Heutigen durch literarische, philosophische und publizistische Schilderungen und zeitgenössische Dokumente darstellt. Das in den Opern Rossinis, Bellinis und Donizettis manifestierte Gesellschaftsbild steht dabei im Zentrum der Betrachtung und wird als künstlerisch und ideologisch gleichermaßen avancierter und effizienter Beitrag zum geistig-weltanschaulichen Diskurs der Zeit verstanden.

Bevor ich die Ergebnisse meiner Studien darlege, möchte ich noch der angenehmen Verpflichtung nachkommen, allen Menschen, die dieses Projekt mit ihrer Tatkraft und Solidarität, mit ihrem Engagement und ihrer Anteilnahme unterstützt haben, meinen aufrichtigen und herzlichen Dank auszusprechen, insbesondere meinem Doktorvater Professor Dr. Jürgen Schläder, den Mitarbeiterinnen und Mitarbeitern des Institutes für Theaterwissenschaft der Ludwig-Maximilians-Universität München und der Fondazione Rossini in Pesaro, insbesondere Herrn Paolo Fabbri, Herrn Professor Bruno Cagli, Herrn Professor Philip Gossett von der University of Chicago, Herrn Professor Friedrich Lippmann vom deutschen historischen Institut in Rom, Herrn Reto Müller von der deutschen Rossini-Gesellschaft, Herrn Capitaneo vom Centro di studi donizettiani in Bergamo, Herrn Dr. Arthur Scherle (+) und Herrn Robert Didion vom Verlagshaus Ricordi, sowie Maestro Marcello Viotti und Maestro Alberto Zedda, die meine Betrachtungen um wertvolle Hinweise aus musikologischer und aufführungspraktischer Sicht ergänzt haben. Ohne den intensiven Austausch und die Hilfen bei Recherche und 
Materialsammlung, die ich den genannten und weiteren Personen zu danken habe, hätte manche gedankliche Brücke nicht geschlagen werden können. 